\title{
Molecular mobility in polymer thin films
}

\author{
K. C. Tseng, N. J. Turro, and C. J. Durning \\ Department of Chemical Engineering and Applied Chemistry, Columbia University, New York, New York 10027
}

(Received 3 September 1998; revised manuscript received 4 August 1999)

\begin{abstract}
Fluorescence recovery after photobleaching was used to measure in-plane dye-probe diffusion coefficients, $D$, in thin films of monodisperse polystyrene supported on fused quartz substrates. The substrates were prepared with a high density of surface hydroxyl groups which interact favorably with repeat units of the polymer. The effects of temperature and film thickness were investigated, at temperatures above the bulk glass transition of the polymer, $T_{g}$, and in the range of film thicknesses from $1-10^{2}$ times the radius of gyration $\left(R_{g}\right)$ of individual polymer molecules. As the film thickness decreases towards $R_{g}$ the value of $D$ increases above the bulk values, with significant effects first appearing in films $\simeq 20 R_{g}$. In the thinnest films studied, about $4 R_{g}$, the values of $D$ lie as much as two orders over bulk values. At the same time, the temperature dependence of $D$ becomes much weaker than in bulk. Analysis by free volume theory indicates that apparent values of both $T_{g}$ and the thermal expansion coefficient for liquid state, $\alpha_{L}$, decrease as the film thickness decreases. The possible effects of surface segregation of the dye probe are discussed.
\end{abstract}

PACS number(s): 61.25.Hq, 68.15.+e, 68.35.Fx, 68.60.-p

\section{INTRODUCTION}

Thin supported polymer films have received much attention recently because in last decade they have been employed increasingly in the rapidly expanding microelectronics industry, as part of the trend in device miniaturization. Molecular mobility in such layers is of broad interest because of its relevance to many performance and processing issues. For instance, molecular diffusion and mobility play key roles in high-resolution photolithography for making integrated circuits, in lubrication for magnetic information storage devices, in the behavior of liquid-crystal displays, and in the application of microelectronics encapsulants and dielectrics [1]. Molecular mobility in thin polymer layers also plays an important role in many other polymer technologies through the processes of wetting, adsorption and adhesion [2].

In microelectronics applications, film thickness becomes an important parameter which varies on a scale comparable to the size of the polymers themselves. As the films become thinner, confinement eventually perturbs the average conformation of the polymer molecules and the density, which can affect molecular mobility and the layer's average properties, including glass transition, thermal expansion, and mechanical properties. However, there are contradictions (vide infra) in experimental results reported so far, and no existing theoretical framework capable of explaining the dynamic properties of thin polymer films, as observed to date. There is clearly a need for more basic work to establish unambiguously how dynamic features differ from the bulk as the film dimensions approach the polymer molecule's radius of gyration, $R_{g}$.

This work uses the holographic grating technique for the measurement of translational diffusion coefficients. The effort focuses on a relatively simple system, consisting of thin films in the melt state of monodisperse, flexible, linear polymer on smooth solid surfaces interacting with the polymers by favorable nonbonding, local interactions (e.g., London dispersion forces and permanent dipole interactions). The principal goal is to systematically explore the effects of film thickness on molecular mobility by a study of smallmolecule tracer diffusion coefficients.

\section{PRIOR WORK}

There is a need to review recent work in some detail because of the lack of consistency in reports so far. There have been several recent studies of polymer chain mobility in thin films. Reiter $[3,4]$ reported indirect evidence of a dependence of chain mobility on polymer film thickness, by x-ray reflectometry studies of the stability of thin polystyrene (PS) films on "float" glass substrates. For films thinner than the average end-to-end distance of the polymer molecules, $R_{E E}$, the average density decreased with film thickness. The reduction of density enhanced mobility, which Reiter detected by the onset of dewetting (i.e., the breakup of a contiguous thin film into droplets) at temperatures much lower than for thicker films. For very thin films $(\$ 5 \mathrm{~nm})$ dewetting was observed even below the bulk glass transition temperature, $T_{g}$. Only slight changes in the dewetting temperatures were observed with molecular weight.

Frank et al. [5] directly measured chain mobility in thin melt PS films supported on quartz by fluorescence recovery after photobleaching (FRAP). The in-plane diffusion coefficient of fluorescently labeled polymer $\left(M_{w}=31 \mathrm{~K}, R_{g}\right.$ $\simeq 3 \mathrm{~nm}$ ) determined by FRAP was substantially lower in thin films than the bulk value, in apparent contradiction with $\mathrm{Re}$ iter's findings. At $140^{\circ} \mathrm{C}$, the polymer diffusion coefficients started to decrease at film thicknesses below about 25 molecular diameters $(\simeq 150 \mathrm{~nm})$. Subsequent discussions suggested that the long-range effect of the surfaces might be caused by tethered chains [6] or by a glass transition shift of uncertain origin [7].

Using secondary ion-mass spectrometry (SIMS), Zheng et al. [8] studied interdiffusion in melts of PS and deuterated PS (dPS) normal to a surface and $\simeq 5 \mathrm{~nm}$ away, for several different surfaces. The process was strongly affected by sur- 
face chemistry. For example, the interdiffusion coefficients on an attractive $\mathrm{SiO}_{n}$ covered silicon surface were smaller by a factor $\sim 100$ than the value near a free surface. They also studied interdiffusion as a function of distance from an attractive $\mathrm{SiO}_{n}$ surface [9]. Long-range effects were observed, reminiscent of those reported by Frank et al. [5]; the diffusion coefficients were as much as an order of magnitude lower than bulk values for distances $\simeq 10 R_{g}$ from the surface.

Lin et al. [10] recently carried out a similar study of interdiffusion in melts of hydrogenated and deuterated poly(methyl methacrylate) (PMMA and dPMMA) near attractive $\mathrm{SiO}_{n}$ surfaces using neutron reflectometry. They also found effective diffusion coefficients much smaller than the bulk values, however, the range of the substrate's effect was only $\sim(3-4) R_{g}$, significantly less than in the studies by Frank et al. [5] and Zheng et al. [8,9].

There are a number of recent studies of the thermal properties of polymer thin films complementing the chain mobility measurements just described. Changes of thermal properties indirectly reflect differences in the dynamic properties; increases in $T_{g}$ or density generally imply slower dynamics.

Using ellipsometry, Keddie et al. [11,12] obtained $T_{g}$ in thin supported PS films. For films on $\mathrm{SiH}$ surfaces on the order of the unperturbed size of the polymers in thickness, $T_{g}$ decreased as film thickness fell, while the glass thermal expansivity, $\alpha_{g}$, increased. The results are reminiscent of Reiter's. Since the effects were not strongly dependent on molecular weight, the authors suggested that they were caused by a liquid-like layer at the polymer-free surface characteristic of the glassy state. Subsequent measurements on PMMA films on two different substrates revealed a strong effect of the polymer-substrate interaction [13]. For PMMA films on an attractive $\mathrm{SiO}_{n}$ surface, $T_{g}$ increased slightly with decreasing film thickness, while for PMMA films on an inert $\mathrm{Au}$ surface, $T_{g}$ decreased. A clear explanation for the effect was not proposed.

van Zanten et al. [14] used x-ray reflectivity to study the same PS system that Keddie et al. examined by ellipsometry, but reported different results. For films of PS with $M_{w}$ $=233 \mathrm{~K}\left(R_{g} \simeq 14 \mathrm{~nm}\right)$ on $\mathrm{SiH}$ surfaces, thinner than about $40 \mathrm{~nm}, T_{g}$ appeared to be greatly increased, by at least $60{ }^{\circ} \mathrm{C}$; for films between $40 \mathrm{~nm}$ and about 200-nm thick, $T_{g}$ was still $\simeq 25^{\circ} \mathrm{C}$ higher than the bulk value. The thermal expansion coefficient above $T_{g}, \alpha_{L}$, was also lower than the bulk value. No completely satisfactory explanation of the discrepancy between the two sets of data has been offered yet.

Positron annihilation lifetime spectroscopy (PALS) has been used recently to measure $T_{g}$ within the first $\simeq 10 \mathrm{~nm}$ at the free surface of relatively thick PS films $\left(M_{w}\right.$ $\left.=260 \mathrm{~K}, R_{g}=14 \mathrm{~nm}\right)$ supported on $\mathrm{SiH}[15]$. The results were consistent with bulk, with no surface-induced reduction in $T_{g}$ in the top 10-nm layer. More recently, the same group reported different results [16] on the same system, of $T_{g}$ in PS films on $\mathrm{SiH}$ as a function of film thicknesses $\left(M_{w}\right.$ $=63 \mathrm{~K}$ and $400 \mathrm{~K} ; R_{g}=7 \mathrm{~nm}$ and $18 \mathrm{~nm}$, respectively). $T_{g}$ dropped as film thickness decreased below about $50 \mathrm{~nm}$ for both molecular weights while the thermal expansion coefficients in the glass and liquid states, $\alpha_{g}$ and $\alpha_{L}$ respectively, were less than the corresponding bulk values. The results for thermal expansion coefficients were consistent with van Zanten et al. [14], but not with Keddie et al. [11], while the depression of $T_{g}$ was consistent with Keddie et al., but not with van Zanten et al. The more recent data give evidence of a liquid-like layer at PS free surface: The thickness dependence of the thermal properties was modeled successfully with a layered, composite film having a surface layer about 5-nm thick with a $T_{g}$ significantly lower than the bulk value.

Forrest et al. $[17,18]$ used Brillouin light scattering to measure $T_{g}$ in freely standing PS films $\left(M_{w}=767 \mathrm{~K}\right.$ and $2240 \mathrm{~K}, R_{E E}=57 \mathrm{~nm}$ and $97 \mathrm{~nm}$ ). For film thicknesses less than $R_{E E}, T_{g}$ decreased linearly with decreasing film thickness, with reductions as large as $60^{\circ} \mathrm{C}$. The $T_{g}$ depression was strongly dependent on the molecular weight for freely standing films. Forrest et al. [18] also used ellipsometry to measure $T_{g}$ in PS films confined by one (uncapped) and two (capped) glass slides. For both the uncapped and capped PS films, $T_{g}$ was reduced only slightly $\left(<10^{\circ} \mathrm{C}\right)$ from the bulk value, with only small differences in $T_{g}\left(<4^{\circ} \mathrm{C}\right)$ between uncapped and capped films of the same thickness.

Despite the discrepancies mentioned, the body of work reviewed here shows clearly that the average dynamic properties in thin films in the range $1-100 R_{g}$ in thickness can be influenced strongly by the two bounding surfaces. Free surfaces (i.e., polymer/vacuum or polymer/gas) appear to enhance molecular mobility, as manifest by suppression of the film's apparent $T_{g}$. This has encouraged the speculation of a highly mobile, low density layer near a free surface that contributes to a drop in a film's apparent $T_{g}$ as the thickness falls towards $R_{g}$. Qualitatively the same picture seems to apply for neutral or weakly interacting solid-melt interfaces. At solid surfaces attractive to polymer, direct measurements show that chain mobility is retarded near the surface while the film's apparent $T_{g}$ seems to increase. The range of influence of a free or solid bounding surface on the film properties, as well as the possible molecular weight dependence of these effects, are still poorly defined by the existing body of experimental work.

There have been a number of theoretical treatments $[19,20]$ and computer simulations [21-29] of mobility and glass transition in polymers confined by walls or near free surfaces [30]. These suggest, at least qualitatively, reasons for enhanced mobility at free surfaces and inert solids, and retarded mobility at attractive walls: (i) an enrichment at free-surfaces of polymer ends, which tends to enhance mobility and lower $T_{g}$ by contributing extra free volume to the surface region; (ii) changes in segment density (i.e., packing) over short length scales (several Kuhn lengths) immediately adjacent to a surface (for free surfaces or neutral walls, the density is suppressed while for attractive surfaces it is enhanced), which alters segmental mobility near the surface, and thereby the overall mobility of the chains with segments at the interface; and (iii) anisotropic segment mobilities near walls (segmental diffusion normal to a surface generally decreases relative to bulk near the surface; diffusion parallel to a neutral surface is enhanced) which also alters mobility of the chains involved.

In what follows, we report the average in-plane translational diffusivity $D$ of a low molecular weight dye probe in thin films of polystyrene (PS) supported on quartz as a function of temperature and film thickness. The technique probes 
the segmental-scale mobility of polymer thin films rather directly. It has not been exploited systematically so far; we are aware of only one prior study of this nature [31], but employing a different method of measurement than discussed here. Our experiments employ supported films on substrates which interact favorably with repeat units of the polymer, so that each film is bounded by a free surface and an attractive solid wall. One can anticipate that the two bounding interfaces will have different, perhaps competitive influences on the average local mobility in a thin film, as revealed by $D$. In particular, we expect the free surface to enhance $D$ while the attractive wall should reduce it.

We find that as the film thickness decreases towards $R_{g}$ the $D$ increase above the bulk values, with effects first appearing in films $\simeq 20 R_{g}$. In the thinnest films studied, about $4 R_{g}$, the $D$ lie as much as two orders above bulk values. The results are qualitatively consistent with thermal property measurements $[11,12,16-18]$ showing a suppression of $T_{g}$ in thin films, although we find significant effects in films much thicker than in these studies.

In the next two sections we describe the experimental technique and results in more detail. Following that, we present an analysis of the diffusivity data in terms of "freevolume'" ideas, which connect $D$ with $T_{g}$, to show our findings correspond to an apparent suppression of $T_{g}$. Finally, we offer an explanation in terms of dye segregation of why we find noticeable elevations in $D$ in films far thicker than those where thermal property shifts first occur in several prior studies.

\section{EXPERIMENT}

\section{A. FRAP Method}

Fluorescence recovery after photo-bleaching (FRAP) is an optical technique for measuring the self or tracer diffusion coefficients of molecules labeled with fluorescent dye which can be irreversibly photo-bleached by intense light. Initially, the dye-labeled molecules are distributed uniformly throughout the sample at a very low concentration. An initial "writing' step creates a spatial variation of fluorescent dye concentration in the sample by an intense burst of light from a laser, which destroys the dye in well-defined regions and thereby eliminates or greatly reduces fluorescence from these regions. A second "reading" operation is detection of fluorescence intensity in the photo-bleached areas. After writing, the sample is illuminated at the same wavelength, on the same region, by a greatly attenuated reading beam; the measured fluorescence intensity gradually recovers as a result of transport of labeled molecules from unbleached parts of the sample into the bleached regions. The rate of recovery can be analyzed for the tracer diffusion coefficients of the labeled species, as discussed below.

In our case, illumination of the sample for both writing and subsequent reading is done with a spatially periodic fringe pattern produced by the interference of two coherent lasers intersecting on the sample. After writing, the pattern is monitored by the same fringe pattern with a greatly reduced intensity. If the dye species translate by ordinary diffusion, the dominant Fourier mode in the transient grating disappears exponentially. The time constant of decay depends inversely on the diffusion coefficient of the labeled molecules and on the square of the diffusion path length. The technique is particularly well suited for the range of diffusion coefficients expected in our system, and for the thin film geometry involved.

We employed a refinement of the fringe pattern technique developed by Davoust et al. [32], exploiting a periodic phase difference between the written and reading fringe patterns, created by a piezoelectrically driven mirror in the optical path of one reading beam. During the recovery, the reading pattern is oscillated over the bleached pattern at a fixed frequency. The resulting fluorescence intensity varies periodically as the illumination stripes alternately fall into and out of phase. The decay envelope of the modulated signal can be extracted from the total measured fluorescence by a lock-in amplifier which suppresses contributions from noise not correlated to the modulation frequency, such as those caused by mechanical vibrations of optical components, dark current of the photodetector, and background fluorescence. One extracts the diffusion coefficient from the rate of decay of the modulation envelope.

\section{Data analysis}

Since part of our discussion involves analysis of fluorescence recovery, we outline the derivation of the exponential decay law for homogeneous samples. During writing or reading, two nearly plane-wave beams of matched intensity, with the wave vectors $\mathbf{k}_{1}$ and $\mathbf{k}_{2}$, intersect at the sample with angles $\pm \theta$ relative to the sample's normal ( $z$ axis). The "'grating", vector, $\mathbf{k}_{0}=\mathbf{k}_{2}-\mathbf{k}_{1}$, lies parallel to the $x$ axis in the sample $(x-y)$ plane. The resulting time-averaged intensity at the sample is

$$
I=I^{0}\left[1+\cos \left(\mathbf{k}_{0} \cdot \mathbf{r}+\phi\right)\right]=I^{0}\left[1+\cos \left(k_{0} x+\phi\right)\right],
$$

where $I^{0}$ is the average light intensity, $\mathbf{r}$ is position measured from an origin in the sample plane, and $k_{0}$ is the spatial frequency of the fringe pattern; $k_{0}=\left|\mathbf{k}_{0}\right|=2 \pi / d$ $=4 \pi \sin \theta / \lambda$. Hence a sinusoidal intensity variation results along the $x$ direction; the period of the intensity variation, i.e., the grating spacing $d$, corresponds to the diffusion distance $d=\lambda /(2 \sin \theta)$.

The initial concentration distribution of fluorescent molecules immediately after photobleaching depends on the photobleaching kinetics as well as on the duration and intensity of the writing beam. Assuming a first-order bleaching reaction

$$
c(\mathbf{r}, 0)=c_{0} \exp \left[-\alpha I_{b}(\mathbf{r}) \Delta t\right]=c_{0} \exp \left\{-K\left[1+\cos k_{0} x\right]\right\},
$$

within the illuminated volume $V$; the second equation results by inserting Eq. (1) (with $\phi=0$ ) for $I_{b}(\mathbf{r}) . c_{0}$ is the prebleach dye concentration (assumed uniform), $\alpha$ is the firstorder rate constant, $I_{b}(\mathbf{r})$ is the bleaching beam intensity, and $\Delta t$ is the duration of bleaching. A bleaching efficiency index, or "'depth of bleach," $K=\alpha I_{b}^{0} \Delta t$, appears as a key parameter.

After photobleaching, the time dependent dye concentration profiles are governed by

$$
C\left(q_{x}, t\right)=C\left(q_{x}, 0\right) \exp \left(-D q_{x}^{2} t\right)
$$


where $q_{x}$ is the wave vector corresponding to $x$ and $C\left(q_{x}, 0\right)$ is the Fourier transform of $c(x, 0)$ [see Eq. (2)]; $D$ is the (tracer or self) diffusion coefficient of the labeled species. The total fluorescence emission from the sample during reading with an intensity profile $I_{m}(\mathbf{r}, t)$ is

$$
F(t)=Q \int_{V} d \mathbf{r} c(\mathbf{r}, t) I_{m}(\mathbf{r}, t),
$$

where $Q$ is an instrument constant; the integral is bounded by the illuminated volume. For fringe-pattern photobleaching, both $c(\mathbf{r}, t)$ and $I_{m}(\mathbf{r}, t)$ depend only on $x$ (a coordinate in the sample plane). Consequently, one can integrate immediately in Eq. (4) along $y$ (the indifferent in-plane direction) and $z$ (the sample-thickness direction); we integrate over a characteristic in-plane size of $V$ along $y, h$, and over the sample thickness $l$ along $z$. The remaining integral, along $x$, can be expressed in terms of modified Bessel functions of the first kind, of order $n, I_{n}(x)$ [33], consequently, the fluorescence emission from $V$ can be written as

$$
F(t)=Q I_{m}^{0} h^{2} l c_{0}\left[A_{0}(K, 0)+A_{1}(K, 0) \exp \left(-D k_{0}^{2} t\right) \cos \phi\right],
$$

where $A_{n}(K, 0)=\exp (-K)(-1)^{n} I_{n}(K)$.

The measured intensity consists of a constant background term and a time-dependent relaxing term. Since the relaxation amplitude $A_{1}(K, 0)$ is typically small compared to the constant term $A_{0}(K, 0)$, we apply a lock-in technique as a signal filter. The phase of the reading fringe pattern is shifted by sinusoidal vibration of a mirror in the optical path. The reading fringes oscillate over the written fringes with a phase around $\phi_{0}$, angular frequency $\omega$ and amplitude $\psi$.

$$
\cos \phi(t)=\cos \left(\phi_{0}+\psi \sin \omega t\right) .
$$

To find the amplitude of the signal $S_{m}(t)$ picked up by the lock-in amplifier at frequency $m \omega$, one expands Eq. (6) in a Fourier series; it turns out that

$S_{m}(t) \propto \begin{cases}A_{1}(K, 0) J_{m}(\psi) \sin \phi_{0} \exp \left(-D k_{0}^{2} t\right) & \text { for } m=\text { odd } \\ A_{1}(K, 0) J_{m}(\psi) \cos \phi_{0} \exp \left(-D k_{0}^{2} t\right) & \text { for } m=\text { even } .\end{cases}$

In our experiments, we chose detection at $2 \omega$, and therefore adjusted $\phi_{0}$ to 0 and set $\psi \simeq 3.1$ to maximize $J_{2}(\psi)$. These adjustments were checked using a sample with immobilized dye, e.g., dye in a tightly cross-linked resin or a glassy polymer sample, where no diffusion takes place on the observation time scale.

\section{Apparatus}

The FRAP instrument in our laboratory is essentially the same as that of Davoust et al. [32]. It consists of a laser light source for writing and subsequent reading, excitation optics including an attenuation system for rapid switching from writing to reading intensity, a sample holder enabling control of the sample temperature, a detection system including fiber-optic collection of emission intensity, a photomultiplier tube, and a lock-in amplifier. The entire setup is mounted on an optical table for isolation against mechanical vibration. A summary of the key components follows. (i) Light source. We employed the 488-nm line of an argon-ion laser (Coherent Innova 70), equipped with an etalon assembly. Typically, the bleaching power was $170 \mathrm{~mW}$ and the bleaching time varied from $100 \mathrm{msec}$ to $3 \mathrm{sec}$. The reading (observation) power was typically about $20 \mu \mathrm{W}$.

(ii) Excitation optics. An attenuation system sits first in the optical train, providing coaxial high and low intensity beams for writing and reading. The intensity ratio between reading and writing beams can be varied from $1 \times 10^{3}$ to $1 \times 10^{7}$. After the attenuation system, a 50/50 beam splitter splits the laser into two coherent beams which are directed by mirrors to recombine on the sample. One mirror can oscillate to facilitate lock-in detection. The set-up allows different crossing angles by moving the oscillatory mirror.

(iii) Sample holder. A sample chamber provides temperature control in the range of $30^{\circ} \mathrm{C}$ to $180^{\circ} \mathrm{C}\left( \pm 1^{\circ} \mathrm{C}\right)$. The entire chamber is Teflon clad, except for an open slot, to prevent the heat loss. The front side allows the optical fiber to reach very close to the sample, to collect a maximum amount of fluorescence emission.

(iv) Detection, control, and data acquisition systems. Fluorescence from the sample is measured with a photomultiplier tube (PMT) (Oriel 77340). An interference filter in the front of the PMT (Oriel 52710; 532 $\pm 10 \mathrm{~nm}$ ) blocks background and scattered radiation, passing only the fluorescence signal from the dye (centered near $532 \mathrm{~nm}$ ). A glass fiber bundle (Dolan-Jenner BXMY2724) transmits the emission from the sample to the PMT. After photobleaching, the oscillatory mirror is activated at frequency $\omega$ by a piezoelectric modulator (Burleigh PZ-91), driven by linear high voltage amplifiers (TREK 601B-3). The driving amps receive input from the lock-in amplifier (LIA) (Stanford Research SR-830). The output current from the PMT is fed back to LIA for filtering and picked up by a data acquisition board. LabVIEW software (National Instruments) controls the experiment, and records and analyzes the data.

\section{B. Materials}

\section{Fluorescent dye}

Rubrene (Aldrich, $M=532$ ) was selected for this work because it has been used successfully in FRAP measurements of tracer diffusion coefficients in bulk PS [34]. To check the thermal stability of rubrene, samples of relatively thick spin-cast polystyrene films with a nominal rubrene concentration of $0.3 \% \mathrm{w} / \mathrm{w}$ were heated to $150{ }^{\circ} \mathrm{C}$ for $6 \mathrm{~h}$ in a vacuum oven $\left(\simeq 10^{-2}\right.$ Torr $)$. The fluorescence spectra of asspun and heated samples were compared and found to be identical in the neighborhood of the laser frequency used. The results indicate that rubrene has good thermal stability, and can be used to study the temperature dependence of tracer diffusion coefficients for temperatures up to $150^{\circ} \mathrm{C}$.

We also checked the effect of the purity of rubrene on the fluorescence characteristics. The as-received dye was purified by recrystallization in toluene. We compared the UV visible spectra, fluorescence excitation and emission spectra and fluorescence lifetime ( $\simeq 14 \mathrm{~ns})$ of the purified and unpurified rubrene solutions, and found no significant differences. Consequently, we used the as-received dye in our experiments. 
TABLE I. Thicknesses by ellipsometry of PS films from spin casting on hydrophilic $(\mathrm{SiOH})$ substrates.

\begin{tabular}{cr}
\hline \hline wt.\% of PS in solution & $l(\mathrm{~nm})$ \\
\hline 0.2 & $9 \pm 1$ \\
0.6 & $36 \pm 2$ \\
1.0 & $55 \pm 3$ \\
1.4 & $118 \pm 5$ \\
1.8 & $178 \pm 5$ \\
2.2 & $230 \pm 5$ \\
2.6 & $292 \pm 7$ \\
3.0 & $339 \pm 7$ \\
5.0 & $424 \pm 7$ \\
10.0 & $930 \pm 20$ \\
\hline \hline
\end{tabular}

\section{Polymer and substrate}

Monodisperse polystyrene (PS) $\left(M_{n}=347000\right.$, polydispersity 1.06 and $R_{g} \simeq 17 \mathrm{~nm}$ ), from Polymer Source, Inc. (Montreal, Canada), was used for all the experiments discussed here. Flat, fused quartz plates $(2$ in. $\times 2$ in. $\times 0.059$ in., Heraeus Amersil 52615, polished SUPRASIL 3, flatness: 10 waves per inch at $633 \mathrm{~nm}$ ) were used as solid substrates. Before contacting the polymers with the quartz plates, the substrates were cleaned and rendered hydrophilic. For cleaning, the substrates were immersed in aqua regia (3:1 concentrated hydrochloric acid: concentrated nitric acid) for $6 \mathrm{~h}$ followed by a rinse with millipore filtered, deionized (18.2 $\mathrm{M} \Omega$ ) water. Then, the plates were oxidized in UV ozone plasma (Jelight Company, Inc., Model 342) for $2 \mathrm{~h}$. The oxidation steps were followed by immersion in concentrated hydrochloric acid for two hours to hydroxylate the surface. The surface was finally rinsed with millipore filtered, deionized water to yield a clean hydrated silanol surface [35-37].

\section{Sample preparation and characterization}

The PS was dissolved in toluene to prepare a series of polymer solutions with concentrations from $0.2 \%$ to $10 \%$ w/w. Rubrene dye was added into the solutions to a nominal concentration of $10^{-4} M$. The solutions were stirred slowly for $48 \mathrm{~h}$, and subsequently filtered through PTFE membranes of pore size $0.45 \mu \mathrm{m}$ (Micron Separations, Inc., Cameo 25F). A small amount $(\simeq 1 \mathrm{ml})$ of the resulting dye/polymer solution was spun onto cleaned quartz plates at $3000 \mathrm{rpm}$ for 60 sec (Headway Research, EC101DT-R485). The resulting films were annealed in a vacuum oven at $150{ }^{\circ} \mathrm{C}$ for $2 \mathrm{~h}$ to dry and relax residual stress.

Dry film samples were characterized by ellipsometry $(\mathrm{Ru}-$ dolph Instruments, Inc., Model 444A12) at room temperature. The wavelength of the incident laser was $632.8 \mathrm{~nm}$, and the angle of incidence was $70^{\circ}$. For simplicity, the compensator azimuth was set so that its fast axis is at $\pm 45^{\circ}$ to the plane of incidence. The film thicknesses of PS films on the hydrophilic $(\mathrm{SiOH})$ surfaces are shown in Table I. Each datum and associated uncertainty was obtained by averaging results of six measurements on the same sample.

\section{Procedure}

Thin film samples were first heated to $160^{\circ} \mathrm{C}$, and then brought down to the experimental temperature to carry out

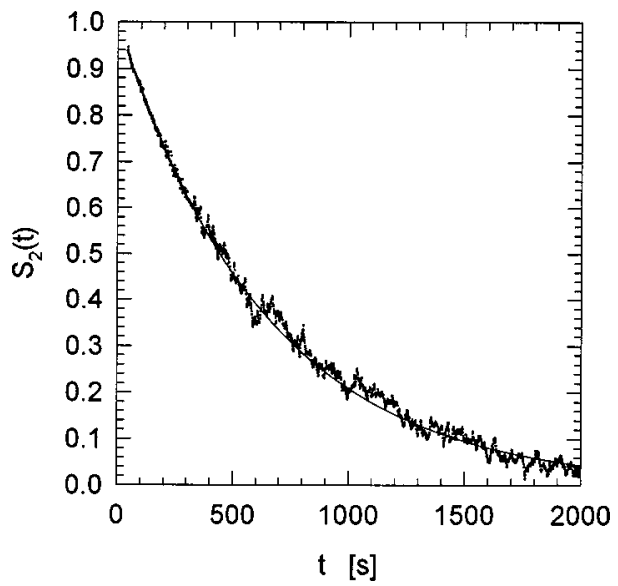

FIG. 1. A typical FRAP decay curve showing the second harmonic amplitude, $S_{2}(t)$, vs decay time, $t$, for rubrene in a PS film $(\simeq 1-\mu \mathrm{m}$ thick $)$ on an $\mathrm{SiOH}$ substrate at $145^{\circ} \mathrm{C}$.

FRAP measurements. During photobleaching, a shutter just before the PMT remains closed to protect the detector from strong fluorescence, and the oscillating mirror stays fixed. After bleaching, the incident beam is attenuated, the shutter protecting the PMT is opened, and the oscillating mirror starts to act. The fluorescence is detected by the PMT and then filtered by the lock-in amplifier.

The recovery curve decays exponentially to zero if the dye transport is diffusive. Curve fitting gives a characteristic time $\tau$ and the diffusion coefficient can be determined by Eq. (8),

$$
D=\frac{1}{\tau k_{0}^{2}} \text {. }
$$

Figure 1 shows a typical FRAP decay curve for rubrene in a thick $(\simeq 1 \mu \mathrm{m})$ PS film. The data were measured at $145^{\circ} \mathrm{C}$ $\pm 1{ }^{\circ} \mathrm{C}$, above the bulk glass transition temperature $T_{g}$ $\left(\simeq 100{ }^{\circ} \mathrm{C}\right)$. In this case the fringe spacing $d$ was $13.2 \mu \mathrm{m}$, the bleaching power was $\simeq 170 \mathrm{~mW}$ and the bleaching time was $500 \mathrm{msec}$. The measured signal indeed decays as a single exponential with characteristic time $\tau=549 \mathrm{sec}$, found by curve fitting (solid line in Fig. 1). The diffusion coefficient is $8.04 \times 10^{-11} \mathrm{~cm}^{2} / \mathrm{sec}$ from Eq. (8).

Of course, for diffusion, $\tau$ should be proportional to the square of diffusion distance, $d^{2}$, with a slope $=1 /\left(4 \pi^{2} D\right)$. This important check is shown in Fig. 2 for the case described above. Error bars in $\tau$ were calculated from the standard deviation in the decay time constants in a set of at least six single FRAP runs. A least-squares fit of the data gave the diffusion coefficient $D=(8.0 \pm 0.31) \times 10^{-11} \mathrm{~cm}^{2} / \mathrm{sec}$. In what follows we report diffusion coefficients determined by the regression method illustrated in Fig. 2.

\section{RESULTS AND DISCUSSION}

Figure 3 shows Arrhenius plots of diffusion coefficients $D$ of rubrene dye in PS films on $\mathrm{SiOH}$ surfaces. The results for the thickest films (424 and $930 \mathrm{~nm}$ ) are in good agreement with previous measurements on the same system in bulk [the dashed line, Fig. 3(a)] reported by Ediger et al. [34] The data show non-Arrhenius behavior (concave down on the Arrhen- 


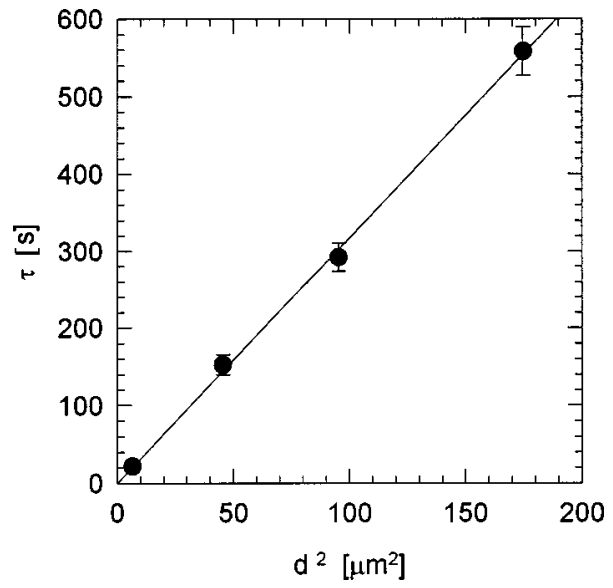

FIG. 2. Decay time constant, $\tau$, vs square of diffusion distance, $d^{2}$, for rubrene diffusion in a PS film $(\simeq 1-\mu \mathrm{m}$ thick $)$ on an $\mathrm{SiOH}$ substrate at $145^{\circ} \mathrm{C}$.

ius plot) typical for transport coefficients in polymer melts above $T_{g}$. The effect of film thickness is striking. In almost every case, for films below about $350 \mathrm{~nm}\left(\simeq 20 R_{g}\right)$, a significant elevation in the diffusion coefficient is observed. Note that the thickness where effects first become significant lies considerably above the depth for the first appearance of significant shifts in the apparent glass transition of supported
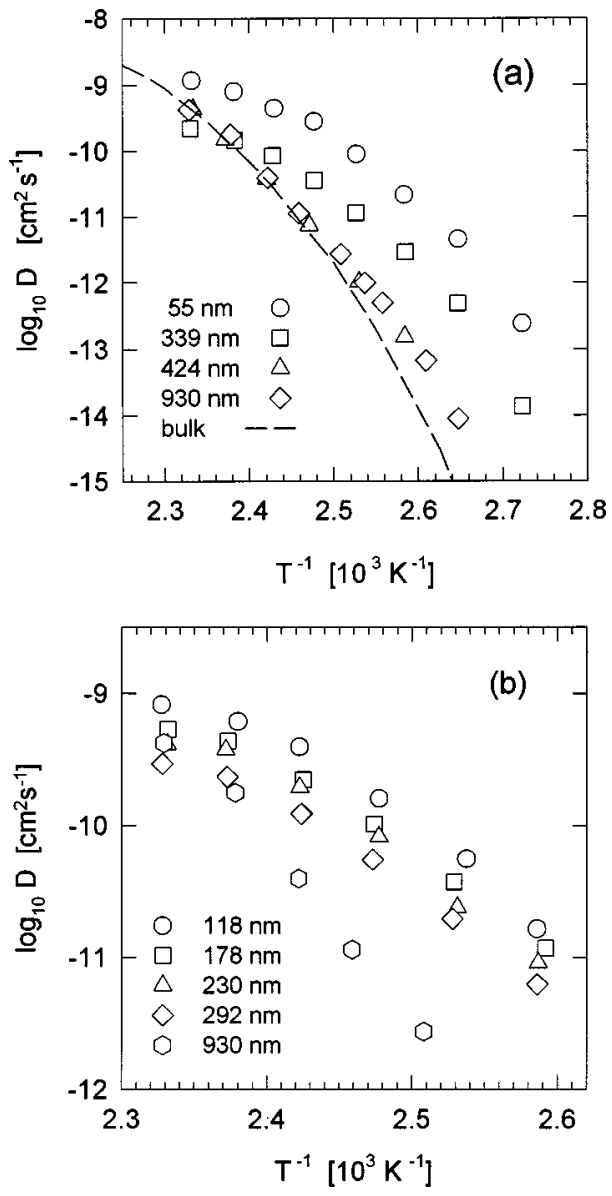

FIG. 3. $\log _{10} D$ vs $1 / T$ for rubrene in PS films on $\mathrm{SiOH}$ substrates for film thicknesses (a) 55, 339, 424, and $930 \mathrm{~nm}$ and (b) $118,178,230$, and $930 \mathrm{~nm}$. For the PS comprising the films, $R_{g}$ $\simeq 17 \mathrm{~nm}$.
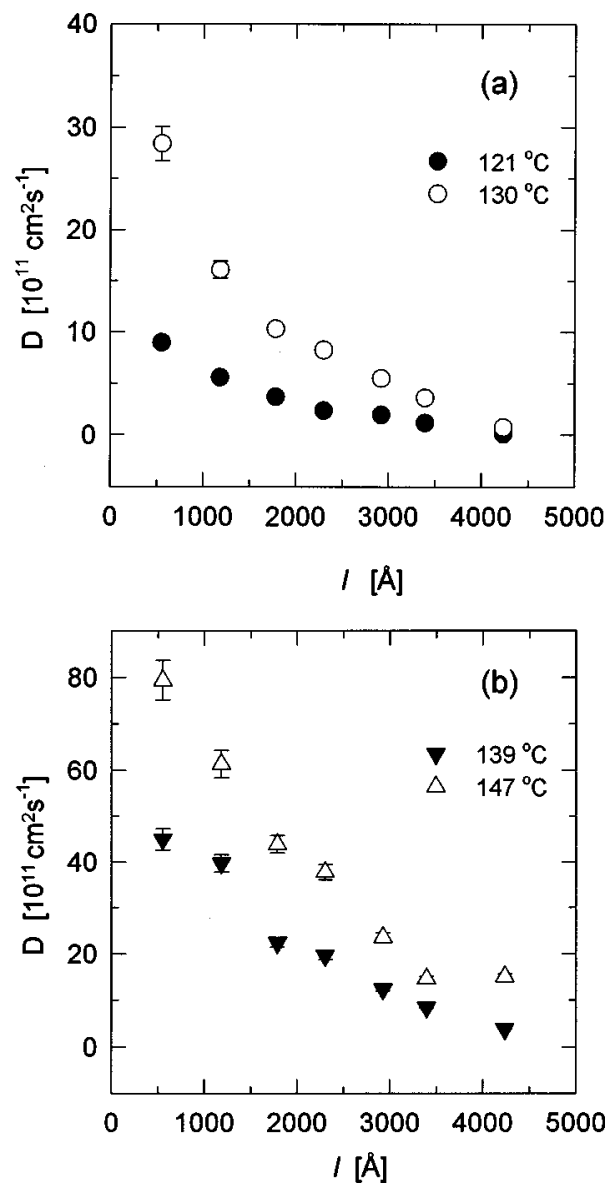

FIG. 4. Diffusion coefficients $D$ of rubrene in PS films on $\mathrm{SiOH}$ substrates vs film thickness, $l$ at (a) $121^{\circ} \mathrm{C}$ and $130^{\circ} \mathrm{C}$ and (b) $139^{\circ} \mathrm{C}$ and $147^{\circ} \mathrm{C}$.

PS films, according to prior work $[11,12,16-18]$. As the film thickness falls below $\simeq 350 \mathrm{~nm}$, the diffusion coefficients increase significantly, by as much as two orders. At the same time the temperature dependence changes, becoming much less severe. Overall the data give the impression that in thin films, the melts become more liquidlike, and less glasslike. It is also notable that for some of the thin films, the diffusivities fall below the bulk values at the highest temperatures studied, i.e., the plots for thin films cross those for thick films towards the ordinate of the Arrhenius plots. Specifically, above $150{ }^{\circ} \mathrm{C}$, we found the diffusion coefficients in some films [339 nm in Fig. 3(a) and $292 \mathrm{~nm}$ in Fig. 3(b)] smaller than the bulk value.

Figure 4 shows the diffusion coefficients as a function of film thickness $l$ at four different temperatures below $150{ }^{\circ} \mathrm{C}$. These plots show explicitly how rubrene diffusion coefficients increase as $l$ is reduced, that the increasing magnitude is strongly dependent on temperature and that the effect can be two orders of magnitude relative to the bulk PS melts. The instrument resolution prevented us from obtaining good FRAP results for PS films less than 50-nm thick.

Two approaches are used to analyze the results in subsequent sections. First, we apply free volume ideas motivated by the prior work indicating that apparent glass transitions and thermal expansion coefficients shift in supported films as thickness decreases. The view is that the effective glass transition and expansivity of sufficiently thin films are perturbed 
by the bounding surfaces (solid/melt and melt/free-surface), and this alters the free volume available for segmental-scale motions and, hence, the average diffusivity of a small dye probe. The main point is to show that the effects in Figs. 3 and 4 are phenomenologically consistent with suppression of $T_{g}$ and $\alpha_{L}$ without offering any detailed explanation as to why such changes occur. A second approach applies a multilayer model which explicitly recognizes interfacial layers with properties different from the bulk. The main point of this analysis is to explain the observed thickness dependence (Fig. 4), including why we first observe effects in relatively thick films, in terms of segregation of the dye to interfacial layers with properties dramatically different from the rest of the film.

\section{A. Free-volume analysis}

Small molecule diffusion in polymer systems has been successfully described using free-volume theory. It relies on the notion that the motion of a small molecule through a liquid medium is controlled by the amount of free space which can redistribute with energy barriers low relative to $k T$. The free space is dependent on the number and type of molecules in the medium (concentration dependence) and on the thermal expansion characteristics of those molecules (temperature dependence). The theory was first developed for polymer melts by Fujita [38], and later modified by Vrentas and Duda (VD) [39-43]. The VD model has been very successful in correlating the effects of temperature and composition on self, tracer and mutual diffusion coefficients in concentrated polymer solutions and melts. We use it here to interpret changes in tracer diffusion coefficients with film thickness in terms of $T_{g}$ and thermal expansivity shifts.

From the VD free-volume model, the diffusion coefficient $D$ of a low molecular weight tracer species in a polymer melt obeys

$$
D=D_{0} \exp \left(-\frac{\gamma \hat{V}^{*} \xi}{\hat{V}_{F H}}\right),
$$

where $D_{0}$ is a constant, $\gamma$ is a dimensionless "overlap" factor (between $\frac{1}{2}$ and 1), $\hat{V}^{*}$ is the "critical" specific free volume of polymer required for a diffusive jump of a freely mobile "jumping unit" on the polymer chain, $\xi$ is the ratio of the critical molar volume of the tracer's jumping unit to that of the polymer, and $\hat{V}_{F H}$ is the specific free volume in the melt. $\hat{V}_{F H}$ can be expressed as $\hat{V}_{F H}=K_{12}\left(K_{22}+T\right.$ $-T_{g}$ ), where $K_{12}$ and $K_{22}$ are constants specific to the polymer. We first use the free volume theory to fit the $D$ for thick, bulklike films, thereby determining unknown constants for the system which are expected to be independent of film thickness.

We define $X$ and $Y$ as

$$
X=\frac{K_{12}}{\gamma}, \quad Y=T_{g}-K_{22},
$$

which are known for bulk PS from viscosity-temperature data and have been reported as $X_{\text {bulk }}=5.82 \times 10^{-4} \mathrm{~cm}^{3} / \mathrm{g} \mathrm{K}$ and $Y_{\text {bulk }}=327 \mathrm{~K}$ [44]. Also, the constant $\hat{V}^{*}$ is known for
PS from molecular architecture, $\hat{V}^{*}=0.850 \mathrm{~cm}^{3} / \mathrm{g}$ [45]. Substituting these into Eq. (9), gives a two-parameter fitting function for rubrene tracer diffusion coefficients in bulk PS,

$$
\ln D=\ln D_{0}-\frac{1461 \xi}{(T-327)},
$$

where the unknown parameters $D_{0}$ and $\xi$ have values specific to the rubrene-PS system, and are expected to be independent of film thickness. If the data for the thickest bulklike film in Fig. $4(930 \mathrm{~nm})$ are replotted as $\ln D$ vs. 1461/( $T$ $-327)$, a straight line indeed results. From the intercept and slope, $\ln D_{0}=-11.2 \pm 0.4$ and $\xi=0.74 \pm 0.02$ are found. These are quite in line with values of these constants for other small molecules in PS [46].

Now, since $\hat{V}^{*}, D_{0}$, and $\xi$ are known, Eq. (9) can be used to fit thin-film data by nonlinear regression to determine the $X$ and $Y$ values for each thin PS film. In particular, the fitting equation is

$$
\ln D=\ln D_{0}-\frac{0.85 \xi}{X(T-Y)} .
$$

Changes in $X$ and $Y$ with film thickness can be interpreted in terms of changes in the film's thermal properties, as follows. $K_{12}$ and $K_{22}$ can be interpreted as [40]

$$
K_{12}=\hat{V}\left(T_{g}\right)\left[\alpha_{L}-\left(1-f^{G}\right) \alpha_{c}\right] ; \quad K_{22}=\frac{f^{G}}{\alpha_{L}-\left(1-f^{G}\right) \alpha_{c}},
$$

where $f^{G}=\hat{V}_{F H}\left(T_{g}\right) / \hat{V}\left(T_{g}\right)$, with $\hat{V}\left(T_{g}\right)$ and $\hat{V}_{F H}\left(T_{g}\right)$ being the specific volume and specific free volume of the polymer at $T_{g}, \alpha_{L}$ being the thermal expansion coefficient of the liquid-state polymer; and $\alpha_{c}$ being the thermal expansion coefficient of polymer's occupied volume. To a good approximation, $\alpha_{c} / \alpha_{L} \simeq 0$, that is, the occupied volume is nearly independent of temperature. Then, $X$ and $Y$ become

$$
X \simeq \frac{\hat{V}\left(T_{g}\right) \alpha_{L}}{\gamma} ; \quad Y \simeq T_{g}-\frac{f^{G}}{\alpha_{L}} .
$$

These relations make it possible to estimate values of $T_{g}$ and $\alpha_{L}$ from $X$ and $Y$.

First, let us estimate the value of $f^{G} / \alpha_{L}$. For PS, the free volume at $T_{g}$ is about $2.5 \%$ of the total volume [47,48], i.e., $f^{G} \simeq 0.025$. The value of $\alpha_{L}$ in bulk PS has been reported as $\left(\alpha_{L}\right)_{\text {bulk }}=5.50 \times 10^{-4} \mathrm{~K}^{-1} \quad$ [49]. Thus, $f^{G} /\left(\alpha_{L}\right)_{\text {bulk }}$ $\simeq 45.5 \mathrm{~K}$, consistent with the value of $46 \mathrm{~K}$ determined directly for bulk PS from the definition of $Y$, i.e., $Y_{\text {bulk }}$ $=\left(T_{g}\right)_{b u l k}-f^{G} /\left(\alpha_{L}\right)_{b u l k}$, where $Y_{\text {bulk }}=327 \mathrm{~K}$ [44] and $\left(T_{g}\right)_{\text {bulk }}=373 \mathrm{~K}$. Since $X / X_{\text {bulk }}=\alpha_{L} /\left(\alpha_{L}\right)_{\text {bulk }}, f^{G} / \alpha_{L}$ can be written as $f^{G} / \alpha_{L} \simeq 46 /\left(X / X_{b u l k}\right)$. Therefore, the $T_{g}$ and $\alpha_{L}$ for thin PS films can be expressed in terms of $X$ and $Y$ as

$$
T_{g} \simeq Y+\frac{46}{X / X_{\text {bulk }}} ; \quad \alpha_{L} \simeq\left(\alpha_{L}\right)_{\text {bulk }} \frac{X}{X_{\text {bulk }}} .
$$

Free volume fits of the thin-film data for the parameters $X$ and $Y$ are shown in Fig. 5. The results for $X / X_{b u l k}$ and $Y / Y_{\text {bulk }}$ as a function of film thickness, $l$, are shown in Fig. 


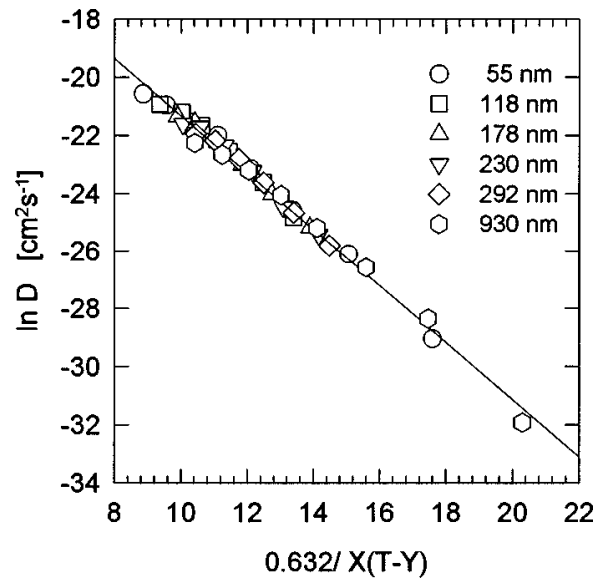

FIG. 5. Analysis of $D$ by free-volume theory for rubrene diffusion in thin PS films on $\mathrm{SiOH}$.

6. Figure 7 shows $\alpha_{L}$ and $T_{g}$ as a function of film thickness according to Eq. (15). The results indicate that both the thermal expansion and glass transition are depressed in thin PS films below bulk values; $T_{g}$ decreases monotonically at first, then levels as $l$ decreases, while $\alpha_{L}$ first decreases and then passes through a weak minimum. The reader should note that the reduction of both $T_{g}$ and $\alpha_{L}$ in a thin film leads to the free volume eventually falling below that in the corresponding bulk as temperature increases. This can explain our observation in Fig. 3 of $D$ in thin films falling below the bulk values above $150{ }^{\circ} \mathrm{C}$.

The results in Fig. 7 are qualitatively consistent with direct thermal property measurements on PS thin films by DeMaggio et al. [16] using positron annihilation (PALS), and by Forrest et al. $[17,18]$ using Brillouin scattering and ellipsometry. In these studies $T_{g}$ was found to decrease with film thickness and in the PALS study, $\alpha_{L}$ also decreased with film thickness. These decreases were associated with the dominant influence of a low density layer at the free surface. Although qualitatively consistent, the results in Fig. 7 differ in detail from these studies in two ways. First, the apparent $T_{g}$ and $\alpha_{L}$ from our data show a plateau or weak minimum as $l$ decreases, which was not seen in references [16-18], and second we notice effects in films much thicker than in the prior work, at $\simeq 350 \mathrm{~nm}$ as opposed to $\simeq 50 \mathrm{~nm}$.

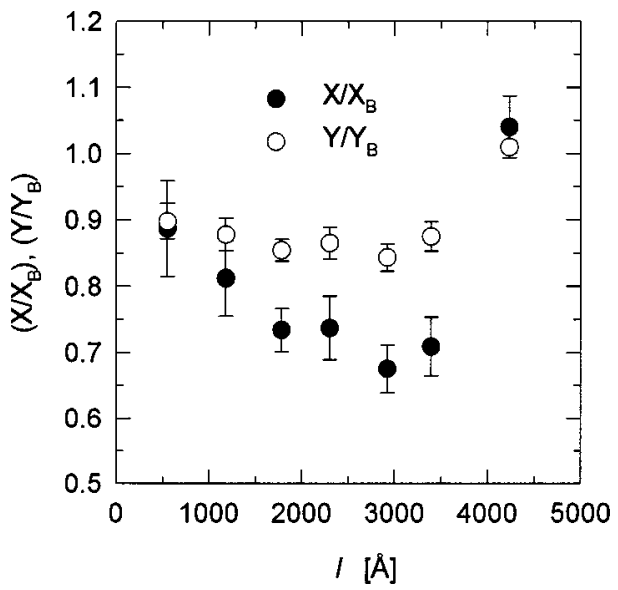

FIG. 6. Parameters $X$ and $Y$ as a function of film thickness for rubrene in $\mathrm{PS}$ films on $\mathrm{SiOH}$.
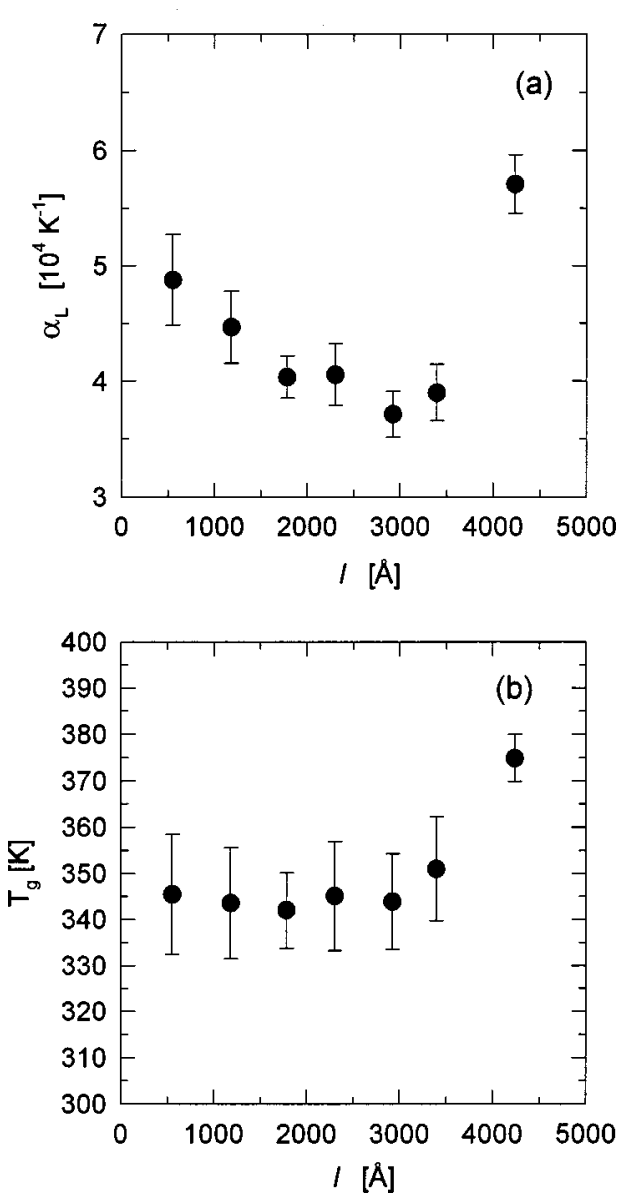

FIG. 7. Apparent values of (a) liquid state thermal expansion coefficients, $\alpha_{L}$, and (b) glass transition temperatures, $T_{g}$, vs film thickness, $l$, for PS films on $\mathrm{SiOH}$.

Regarding the first discrepancy, although a plateau or weak minimum in $T_{g}$ or $\alpha_{L}$ with $l$ might be rationalized [50], it must be acknowledged that we are not reporting direct measurements in Fig. 7 but indirect estimates from $D$ via Eqs. (12)-(15) involving several presumptions and with rather large uncertainties in the final values. In our judgement, one should not utilize the results in Fig. 7 in a testing comparison with prior direct measurements, but rather as a vehicle for qualitative comparisons between the trends we observe in $D$ and those from direct measurements of $T_{g}$ or $\alpha_{L}$. The second discrepancy, concerning the range of influence of the bounding surfaces, demands more careful analysis.

\section{B. Two-layer model}

The majority of prior work on thin films suggests the presence of narrow layers with properties different from the bulk near each surface which influence the observed behavior. In particular, a layer of low density and enhanced mobility is suspected at a free surface or weakly interacting solid, while the opposite is suspected at a strongly attractive surface. From our finding of enhanced dye mobility and suppression of the apparent $T_{g}$ in thin films, we surmise that a mobile layer at the free surface plays the dominant role in this system, and is responsible for the observed effects. Although this seems clear, the range of the free surface's effect, 


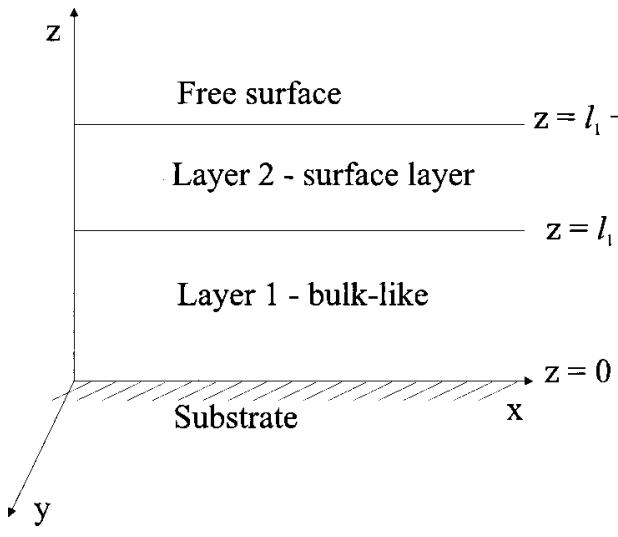

FIG. 8. Schematic of two-layer model.

about $20 R_{g}$, is greater than one would expect from prior work and from the physics involved. One expects a suppression of the total density near the free surface over a length scale on the order of perhaps a few Kuhn lengths (1-3 nm); beyond this one should recover the bulk density. Since it is the local total density which governs the mobility of a small tracer, it is hard to explain the onset of elevated mobilities at $20 R_{g}$ for a homogeneously distributed dye, unless some unexpected long-ranged effects are operating.

There is a possibility, however, that the dye probe is energetically or entropically favored at the free surface and adsorbs to form a concentrated surface layer, strongly influencing the observed mobility in relatively thick films. If the dye mobility at the surface is significantly higher than the bulk, one could see the onset of elevated mobilities in films much thicker than the layer of adsorbed dye, simply because much of the dye contributing to the FRAP signal is concentrated there. Indeed, for our films there is typically not enough dye to form a dense monolayer, so that if strong dye adsorption did occur, most of the dye would be segregated to the surface, and the measured signals would reflect the properties of the surface-segregated layer. Direct verification of this scenario is perhaps possible, but beyond the scope of the present investigation. Instead we consider whether a simple two-layer model accounting for dye segregation into a thin surface layer with enhanced mobility can qualitatively account for appearance of elevated diffusivities in relatively thick films.

Here, the polymer film is modeled as two homogeneous layers, one with high mobility near the free surface and one bulklike, with low mobility between the free and solid interfaces (Fig. 8). The dye partitions between the two layers, which remain in local equilibrium during FRAP. Because of the thin film geometry, the ratio of the film thickness $l$ to the fringe pattern spacing $d$ is small. This allows a perturbation analysis to find the FRAP response (see the Appendix). The important result is that the homogeneous film's diffusivity $D$ in Eq. (7) gets replaced by an effective diffusivity for the two-layer film,

$$
D=\frac{l_{1} D_{1}+\alpha_{12} l_{2} D_{2}}{l_{1}+\alpha_{12} l_{2}},
$$

where $l_{i}$ is the thicknesses of the bulklike $(i=1)$ and surface $(i=2)$ layers, $D_{i}$ is the dye diffusivity in the two layers, and
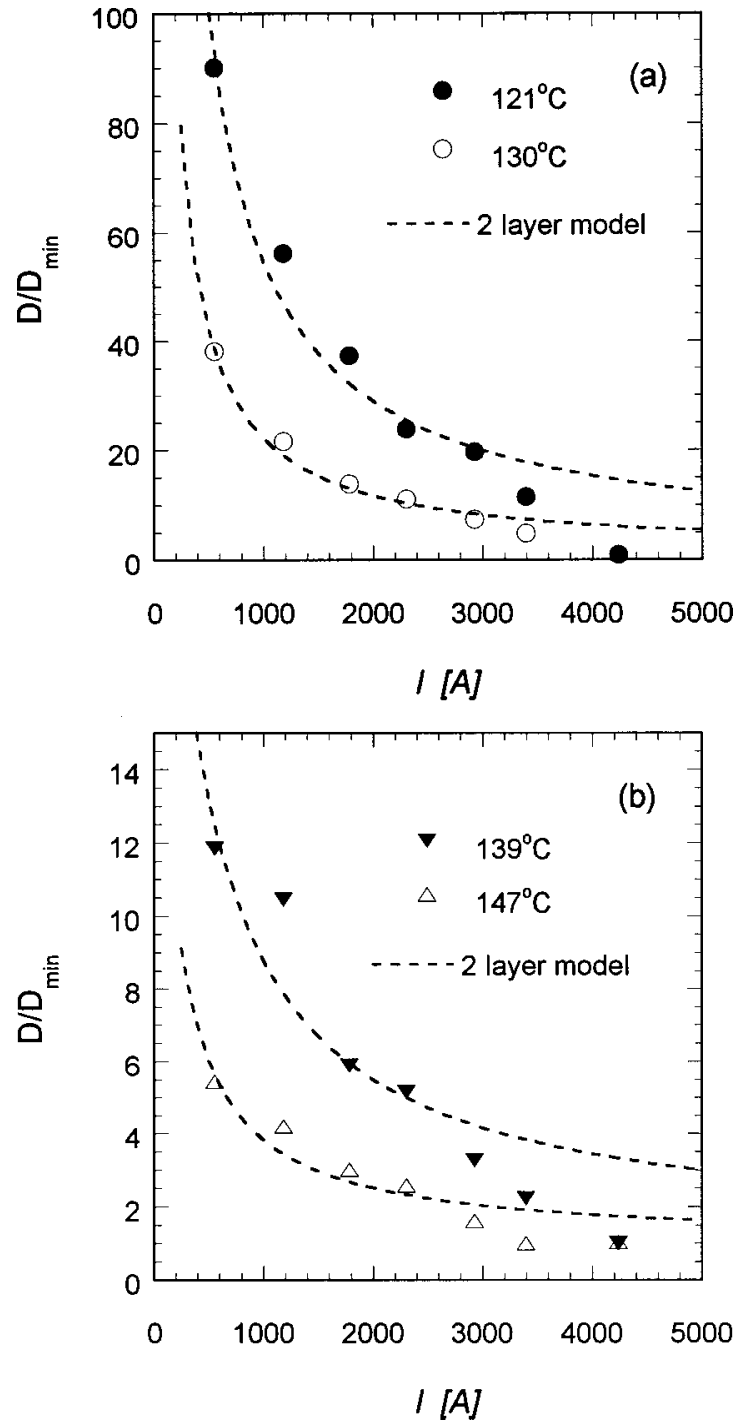

FIG. 9. Regression fits of the two-layer model to $D / D_{\min }$ vs $l$ for rubrene in PS films on $\mathrm{SiOH}$ at (a) $121^{\circ} \mathrm{C}$ and $130^{\circ} \mathrm{C}$ and (b) $139^{\circ} \mathrm{C}$ and $147^{\circ} \mathrm{C}$.

$\alpha_{12}$ is the partition coefficient of the dye between the two layers, expressed as the ratio of dye concentration in the surface layer to that in the bulklike layer at equilibrium.

Equation (16) provides a basis for analyzing the data in Fig. 4, as follows. We expect that the surface layer's thickness, $l_{2}$, is on the order of $1-3 \mathrm{~nm}$, while the total film thickness, $l_{1}+l_{2}=l$, lies in the range $10^{2}-10^{3} \mathrm{~nm}$. Clearly, we can assume $l_{1} \simeq l$ in Eq. (16). Then, dividing Eq. (16) by the diffusivity of the bulklike layer, $D_{1}$, supplies a two parameter fitting function

$$
\frac{D}{D_{1}}=\frac{l+A}{l+B}
$$

where $A=\alpha_{12} l_{2}\left(D_{2} / D_{1}\right)$ and $B=\alpha_{12} l_{2}$. Figure 9 shows regression fits of the data in Fig. 4; the fits are quite reasonable representations. Unfortunately the uncertainties in both $A$ and $B$ are relatively large. Only the order of magnitude of $B$ could be determined; we found $B \sim 10 \mathrm{~nm}$ for all the cases examine $\left(T=121^{\circ} \mathrm{C}, 130^{\circ} \mathrm{C}, 139^{\circ} \mathrm{C}\right.$, and $\left.147^{\circ} \mathrm{C}\right)$. Values 
TABLE II. Parameter $A$ from fits of two-layer model to $D$ vs $l$.

\begin{tabular}{cc}
\hline \hline$T\left({ }^{\circ} \mathrm{C}\right)$ & $A(\mathrm{~nm})$ \\
\hline 121 & $6000 \pm 1200$ \\
130 & $2200 \pm 400$ \\
139 & $1100 \pm 200$ \\
147 & $340 \pm 90$ \\
\hline
\end{tabular}

of $A$ are known to within $\pm 20 \%$; Table II gives the values estimated. Three remarks are relevant.

First, the order of magnitude of $B=\alpha_{12} l_{2}$ suggests significant surface segregation, since if $l_{2} \sim 1 \mathrm{~nm}$, then $\alpha_{12} \sim 10$. Second, note that $A / B=D_{2} / D_{1}$ corresponds to the ratio of mobilities in the surface and bulklike layers; the values are of order $D_{2} / D_{1} \sim 10-100$, consistent with the notion of a surface layer with mobility significantly enhanced over the bulk. Third, we observe a significant trend with temperature in the parameter $A$; it decreases by about an order of magnitude in the $26^{\circ} \mathrm{C}$ interval from $121^{\circ} \mathrm{C}-147^{\circ} \mathrm{C}$. We question if this is reasonable. Assuming that $B$ is independent of temperature in this interval, the majority of the variation in $A$ must come from the temperature dependence of $D_{2} / D_{1}$. An order of magnitude drop in $D_{2} / D_{1}$ is indeed consistent with the free volume expression for $D$ [Eqs. (9) or (12)] if the glass transition and liquid-state expansion coefficient of the surface layer lie considerably below that of the bulklike layer. In particular, assuming a $50{ }^{\circ} \mathrm{C}$ suppression of $T_{g}$ and a 30\% reduction of $\alpha_{L}$ in the surface layer relative to bulk accounts for an order of magnitude drop in $D_{2} / D_{1}$, according to the free-volume theory.

One can say that the two-layer analysis offers a reasonable explanation for the appearance of significant elevations in $D$ in films of $20 R_{g}$ without contradicting results from prior thermal property studies or invoking long-ranged effects of the bounding surfaces.

\section{SUMMARY AND CONCLUSIONS}

We measured dye-probe diffusion coefficients $D$ in PS thin films on hydroxylated quartz $(\mathrm{SiOH})$ substrates. Film thicknesses were in the range $50-1000 \mathrm{~nm}$, and the temperature was varied from $\simeq 110-160^{\circ} \mathrm{C}$. We observe that $D$ increases as film thickness decreases for temperatures below $\simeq 150{ }^{\circ} \mathrm{C}$. The increase is strongly dependent on temperature. Above $150^{\circ} \mathrm{C}$, we found the diffusion coefficients in some films are smaller than the bulk value. An apparent longrange effect of the surfaces has been observed, since the effect on measured mobilities begins at about $20 R_{g}$.

We employed the Vrentas-Duda free volume theory to analyze the data, and find that both thermal expansion $\alpha_{L}$ and the glass transition temperature $T_{g}$ in thin films are lower than bulk values. This result explains why it is possible for the thin-film diffusivities to fall below those in thick films at high enough temperature. The results are qualitatively consistent with dewetting measurements for PS films on float glass [3,4], and $T_{g}$ measurements by ellipsometry for PS films on $\mathrm{SiH}$ surfaces [11,12], by positron annihilation for PS films on $\mathrm{SiH}$ surfaces [16], and by Brillouin light scattering for freely standing PS films and ellipsometry for PS films confined by one or two glass slides $[17,18]$, as well as most of theoretical predictions and computer simulation results which predict the enhanced mobility near a free surface. However, in our experiments significant effects are first noticed in films far thicker than in the studies just cited. To address this issue, we also analyzed the data with a simple "two-layer" model accounting for dye segregation to a free surface layer with enhanced mobility. The model can account for the effects of film thickness we observe with a narrow (1-3-nm) layer near the surface with mobility $\sim 10-100$ times that of the bulk where the dye concentration up to $\sim 10 \times$ that in the bulk. Direct experimental checks of this possibility are in progress.

Of course, the supported thin-film geometry used here only allows study with two interfaces simultaneously present, polymer-solid and polymer free surface, each with different physical and/or chemical attributes. These two interfaces can play separate and perhaps competitive roles in determining the average molecular mobility of a tracer in a thin film. Although prior work suggests that near a free surface there exists a layer where the molecular mobility is much higher than in bulk, it also suggests that near the attractive $\mathrm{SiOH}$ surface, a denser, "bound" layer could be formed, where the mobility is suppressed and glasslike features contributes to average behavior. We have not addressed this issue explicitly in the present work. In effect, our twolayer treatment lumps the influence of both interfaces together. The question of how the polymer-solid interface affects the dye mobility is addressed in another article.

\section{ACKNOWLEDGMENTS}

We thank Professor A. Couzis (City College of New York) for help with ellipsometry measurements, and Professors J. Dhont (University of Utrecht) and J. Thomas (Columbia University) for advice on FRAP setup. This research was supported by the National Science Foundation, Grant No. CTS 9634594.

\section{APPENDIX}

Figure 8 shows a schematic of a two-layer model. The thin film lies in the $x-y$ plane. The periodic fringe-pattern varies along the $x$ direction. The film is heterogeneous along $z$, consisting of a bottom layer with bulklike properties (denoted with subscript $i=1$ ) and an upper layer adjacent to the free surface with different properties (denoted by subscript $i=2$ ). We assume the two layers are in local equilibrium at the interface $z=l_{1}$, i.e., that the dye partitions across the interface according to the law governing phase equilibrium between the bulklike and surface layers.

To determine the response in a FRAP experiment, we need to evaluate Eq. (4). As in the case of a homogeneous layer, one can carry out the $y$ integration immediately. In general, the $z$ integration cannot be done for the two layer film without knowing the $z$ dependence of $c(\mathbf{r}, t)$ explicitly. However, for the case of thin film geometry, where the characteristic diffusion distance in the $x$ direction greatly exceeds the film thickness $l_{1}+l_{2}=l$, the diffusion process is virtually one dimensional, independent of $z$. This can be shown explicitly by a perturbation analysis [51] of dye diffusion in the two-layer film. 
In the heterogeneous film, dye concentrations obey

$$
\frac{\partial c_{i}}{\partial t}=D_{i}\left[\frac{\partial^{2} c_{i}}{\partial x^{2}}+\frac{\partial^{2} c_{i}}{\partial z^{2}}\right]
$$

in each layer. Because of the periodicity of the fringe pattern, the boundary conditions along $x$ are

$$
\left.\frac{\partial c_{i}}{\partial x}\right|_{x=0, d}=0
$$

Along $z$, the boundary conditions are

$$
\begin{gathered}
\left.\frac{\partial c_{1}}{\partial z}\right|_{z=0}=0 ; \quad-\left.D_{1} \frac{\partial c_{1}}{\partial z}\right|_{z=l_{1}}=-\left.D_{2} \frac{\partial c_{2}}{\partial z}\right|_{z=l_{1}} \\
\left.\alpha_{12} c_{1}\right|_{z=l_{1}}=\left.c_{2}\right|_{z=l_{1}} ; \quad-\left.D_{2} \frac{\partial c_{2}}{\partial z}\right|_{z=l_{1}+l_{2}}=0 .
\end{gathered}
$$

The first and the last expressions show that the dye cannot escape the film, the second is mass conservation at the interface $z=l_{1}$, and the third expression shows local equilibrium between the two layers. We presume linear partitioning between the two layers at equilibrium; for surface segregation $\alpha_{12}>1$. The initial conditions for diffusion are

$$
c_{i}(x, z, t=0)=c_{i, 0} h(x),
$$

where $h(x)$ is a smooth periodic function giving the fringe pattern, and $c_{i, 0}$ are the initial, pre-bleach concentrations in the two layers $\left(c_{2,0} / c_{1,0}=\alpha_{12}\right)$.
Scaling the above using a characteristic time for FRAP recovery on a bulk-like layer and the natural characteristic length scales along $x$ and $z$

$$
c_{i}^{*} \equiv c_{i} / c_{i, 0} ; \quad t^{*} \equiv t D_{1} / d^{2} ; \quad x^{*} \equiv x / d ; \quad z^{*} \equiv z / l
$$

gives a dimensionless model for dye transport with one parameter $\varepsilon \equiv(l / d)^{2}$. For all the conditions studied in this work $\varepsilon \ll 1$. Assuming

$$
\left.\begin{array}{l}
c_{1}^{*} \sim a_{0}+\varepsilon a_{1}+0(\varepsilon) \\
c_{2}^{*} \sim b_{0}+\varepsilon b_{1}+0(\varepsilon)
\end{array}\right\} \varepsilon \equiv(l / d)^{2}
$$

we find at lowest order, $O\left(\varepsilon^{0}\right)$ :

$$
\begin{gathered}
a_{0}=b_{0}=A_{0}\left(x^{*}, t^{*}\right) \\
\left(\frac{\partial}{\partial t^{*}}-d \frac{\partial^{2}}{\partial x^{* 2}}\right) A_{0}=0 \\
d \equiv \frac{D}{D_{1}} \equiv \frac{\alpha_{12} l_{2}\left(D_{2} / D_{1}\right)+l_{1}}{\alpha_{12} l_{2}+l_{1}} \\
\left.\frac{\partial A_{0}}{\partial x^{*}}\right|_{x^{*}=0,1}=0 ; \quad A_{0}\left(x^{*}, t^{*}=0\right)=h(x) .
\end{gathered}
$$

Equation (A7) indicates that in the limit of thin films, the dye transport is one dimensional along $x$ with an effective diffusion coefficient $D$, a weighted average of the diffusivities in each layer. The weight factors are proportional to the amount of dye in each layer.
[1] C. W. Frank, V. Rao, M. M. Despotopoulou, R. F. W. Pease, W. D. Hinsberg, R. D. Miller and J. F. Rabolt, Science 273, 912 (1996).

[2] I. C. Sanchez, Physics of Polymer Surfaces and Interfaces (Butterworth-Heinemann, Greenwich, 1992).

[3] G. Reiter, Europhys. Lett. 23, 579 (1993)

[4] G. Reiter, Macromolecules 27, 3046 (1994).

[5] B. Frank, A. P. Gast, T. P. Russell, H. R. Brown and C. Hawker, Macromolecules 29, 6531 (1996).

[6] P. Calvert, Nature 384, 311 (1996).

[7] T. P. Russell and S. K. Kumar, Nature 386, 772 (1997).

[8] X. Zheng, B. B. Sauer, J. G. Van Alsten, S. A. Schwarz, M. H. Rafailovich, J. Sokolov, and M. Rubinstein, Phys. Rev. Lett. 74, 407 (1995).

[9] X. Zheng, M. H. Rafailovich, J. Sokolov, Y. Strzhemechny, S. A. Schwarz, B. B. Sauer, and M. Rubinstein, Phys. Rev. Lett. 79, 241 (1997).

[10] E. K. Lin, W. Wu, and S. K. Satija, Macromolecules 30, 7224 (1997)

[11] J. L. Keddie, R. A. L. Jones, and R. A. Cory, Europhys. Lett. 27, 59 (1994).

[12] J. L. Keddie and R. A. L. Jones, Isr. Jo. Chemi. 35, 21 (1995).

[13] J. L. Keddie, R. A. L. Jones, R. A. Cory, Faraday Discuss. 98, 219 (1994).

[14] W. E. Wallace, J. H. van Zanten and W. L. Wu, Phys. Rev. E 52, R3329 (1995).
[15] L. Xie, G. B. DeMaggio, W. E. Frieze, J. DeVries, D. W. Gidley, H. A. Hristov, and A. F. Yee, Phys. Rev. Lett. 74, 4947 (1995).

[16] G. B. DeMaggio, W. E. Frieze, D. W. Gidley, M. Zhu, H. A. Hristov, and A. F. Yee, Phys. Rev. Lett. 78, 1524 (1997).

[17] J. A. Forrest, K. Dalnoki-Veress, J. R. Stevens, and J. R. Dutcher, Phys. Rev. Lett. 77, 2002 (1996).

[18] J. A. Forrest, K. Dalnoki-Veress, and J. R. Dutcher, Phys. Rev. E 56, 5705 (1997).

[19] R. Bruisnma, Macromolecules 23, 276 (1990).

[20] A. M. Mayes, Macromolecules 27, 3114 (1994).

[21] I. Bitsanis and G. Hadziioannou, J. Chem. Phys. 92, 3827 (1990).

[22] K. F. Mansfield and D. N. Theodorou, Macromolecules 22, 3143 (1989).

[23] D. N. Theodorou, Macromolecules 22, 4589 (1989).

[24] K. F. Mansfield and D. N. Theodorou, Macromolecules 23, 4430 (1990).

[25] K. F. Mansfield and D. N. Theodorou, Macromolecules 24, 6283 (1991).

[26] J. Baschnagel and K. Binder, Macromolecules 28, 6808 (1995).

[27] J. Baschnagel and K. Binder, J. Phys. I 6, 1271 (1996).

[28] K. Binder, A. Milchev and J. Baschnagel, Annu. Rev. Mater. Sci. 26, 107 (1996).

[29] J. Baschnagel and K. Binder, Macromol. Symp. 106, 41 (1996). 
[30] It should be noted that all the simulations referenced are for chains below the entanglement threshold. No computer simulations to date have been able to examine interfacial dynamics above the entanglement molecular weight.

[31] D. B. Hall, R. D. Miller, and J. M. Torkelson, J. Polym. Sci., Part B: Polym. Phys. 35, 2795 (1997).

[32] J. Davoust, P. F. Devaux and L. Leger, EMBO J. 1, 1233 (1982).

[33] Handbook of Mathematical Functions, edited by M. Abramowitz and I. A. Stegun (U.S. Department of Commerce, National Bureau of Standards, Washington, DC, 1970).

[34] M. T. Cicerone, F. R. Blackburn, and M. D. Ediger, Macromolecules 28, 8224 (1995).

[35] R. K. Iler, The Chemistry of Silica (Wiley, New York, 1979).

[36] E. Pefferkorn, A. Haouam, and R. Varoqui, Macromolecules 21, 2111 (1988).

[37] C. J. Durning, B. O’Shaughnessy, U. Sawhney, D. Nguyen, J. Majewski, and G. Smith, Macromolecules 32, 6772 (1999).

[38] H. Fujita, Fortsch. Hochpolymeren Forsch. 3, 1 (1961).

[39] J. S. Vrentas and J. L. Duda, J. Polym. Sci. Polym. Phys. Ed. 15, 403 (1977); 15, 417 (1977).

[40] J.-S. Vrentas and J. L. Duda, J. Appl. Polym. Scie. 22, 2325 (1978).

[41] J. S. Vrentas and J. L. Duda, AIChE. J. 25, 1 (1979).

[42] S. T. Ju, H. T. Liu, J. L. Duda, and J. S. Vrentas, J. Appl. Polym. Sci. 26, 3735 (1981).
[43] J. L. Duda, J. S. Vrentas, S. T. Ju, and H. T. Liu, AIChE. J. 28, 279 (1982).

[44] J. D. Ferry, Viscoelastic Properties of Polymers (Wiley, New York, 1980), 3rd Ed.

[45] R. N. Haward, J. Macromol. Sci., Chem. 4, 191 (1970).

[46] K. C. Tseng, N. J. Turro, and C. J. Durning, Polymer Commun. (to be published).

[47] M. L. Williams, R. F. Landel and J. D. Ferry, J. Am. Chem. Soc. 77, 3701 (1955).

[48] H. Fujita, in Diffusion in Polymers, edited by J. Crank and G. S. Park (Academic, London, 1968).

[49] J. T. Seitz, J. Appl. Polym. Sci. 49, 1331 (1993).

[50] Although the minimum in $\alpha_{L}$ and the leveling of $T_{g}$ with $l$ (Fig. 7) were not seen in direct measurements by DeMaggio et al. [16] and Forrest et al. $[17,18]$, both can be rationalized by the free surface and melt-solid interfaces exerting different influences on the average value of $D$ in thin supported films. The prior work established that a free surface tends to suppress both $T_{g}$ and $\alpha_{L}$ while an interactive solid surface does the opposite, hence the free surface should tend to enhance $D$ while the solid should tend to suppress it. If the two surfaces have unequal, opposing influences, non-monotonic trends in the $T_{g}$ and $\alpha_{L}$ calculated from $D$ could result.

[51] J. Kevorkian and J. D. Cole, Perturbation Methods in Applied Mathematics (Springer-Verlag, New York, 1981). 